\title{
Large Lepton Number of the Universe and The Fate of Topological Defects
}

\author{
Borut Bajc ${ }^{(1,3)}$, Antonio Riotto ${ }^{(2,3)}$ and Goran Senjanović( ${ }^{(3)}$ \\ (1) Department of Physics, University of Durham, Durham DH1 3LE, UK, and J. Stefan Institute, 1001 Ljubljana, Slovenia \\ ${ }^{(2)}$ Department of Physics, Theoretical Physics, University of Oxford, 1 Keble Road, Oxford OX1 3NP, UK \\ ${ }^{(3)}$ International Center for Theoretical Physics, 34100 Trieste, Italy
}

(October 22, 2018)

\begin{abstract}
We show that the monopole problem in Grand Unified Theories as well as the domain wall problem may be easily solved if the lepton number asymmetry in the Universe is large enough.

PACS: $98.80 . \mathrm{Cq} \quad$ DTP/97/92, IJS-TP-97/16, OUTP-97-49-P
\end{abstract}

A. Introduction. Monopole and domain wall problems are some of the central issues in the modern astroparticle physics. The problem of monopoles is especially serious since it is generic to the idea of grand unification [1]. The popular solution based on the idea of inflation cannot be implemented in the minimal Grand Unified Theories (GUTs), and even if it does work it would imply a sad prediction of essentially no monopoles in the Universe, and thus eliminate a prospect of observing this exciting aspect of charge quantization. Of course, it is hard to imagine a Universe without ever having passed through an era of inflation; we simply take here the point of view that this may have happened before the time of grand unification in the thermal history of the Universe. Similarly, the problem of domain walls [2] in theories with a spontaneous breaking of discrete symmetries requires inflation to take place after the phase transitions that cause the production of these defects, which is difficult to achieve in general. Recently, a possible solution of the monopole problem was suggested [3], based on the possibility that unstable domain walls sweep away the monopoles.

There is another possible way out of these problems and it is based on an unusual picture of non restoration of symmetries at high temperatures. It has been known for a long time that in theories with more than one Higgs multiplet, which seems to be a necessary feature of all theories beyond the Standard Model (SM), broken symmetries may remain broken at high temperature $T$ in some regions of the parameter space, and even the unbroken ones may get broken as the system in question is heated up [4,5].

The idea of symmetry nonrestoration provides a simple way out of the domain wall problem [6, ,7], but unfortunately in the case of the monopole problem the situation is far from clear 8 , since next to the leading order effects tend to invalidate this picture for local symmetries [9]. While in the case of discrete symmetries the original lattice calculations spoke against nonrestoration at high $T$ [10], the latest results give full support of this idea [11], as do the other nonperturbative results [12]. However, it can be shown that this scenario does not work in supersymmetry. More precisely, there is a rigorous proof at the renormalizable level 13.14, and the simple counter examples at the non-renormalizable level [15] have been shown not to work [16].

A manifestation of nonrestoration is an old idea 17] of $U(1)_{e m}$ breaking at temperatures above $M_{W}$. Unfortunately, this suffers from the same next-to-leading order effects mentioned above [7]. There is a simple variation of this scenario where $U(1)_{\text {em }}$ is broken only in a very narrow range of temperatures around the electroweak scale [18], [19]. In this case the monopoles get produced with the hope of being annihilated fast enough through the strings attached to monopole-antimonopole pairs. However, there is a serious question whether the annihilation does the job [20], 21].

The situation becomes much more promising if one accepts the possibility of having a large background charge in the Universe, large in a sense of being comparable to the entropy [22]. The presence of some sizeable charge asymmetry may postpone symmetry restoration in nonsupersymmetric theories 23] or, even more remarkably, it can lead to symmetry breaking of internal symmetries at high temperature 24]. Furthermore, the phenomenon of symmetry nonrestoration at high $T$ in presence of large charge asymmetries has been recently shown to work in supersymmetry too 25]. The principal candidate for a large charge is the lepton number which today could reside in the form of neutrinos. This has inspired Linde in his original work to point out that large enough lepton number of the Universe would imply the nonrestoration of symmetry even in the SM [26]. While one could naively think that the large lepton number would be washed out by the sphaleron effects at the temperature above the weak scale, it turns out that the nonrestoration of symmetry prevents this from happening [27], and remarkably enough up to this day this still remains a consistent possibility. Indeed, the successful predictions of primordial nucleosynthesis are not jeopardized as long as the lepton number is smaller than $\sim 7 T^{3}$ at temperatures of the order of $1 \mathrm{MeV}$ [28]. It is therefore natural to ask ourselves whether a large lepton asymmetry in the Universe may play any significant role in solving the monopole problem. The main point we wish to make in this Letter is that the answer may be positive if these two basic requirements are satisfied: the large lepton asymmetry leads to the symmetry nonrestoration of the SM gauge symmetry and some charge field condensation takes place. While it is not clear whether this happens in SM, it is certainly 
true for its minimal extensions (such as an additional charged scalar). Thus, if the lepton number of the Universe were to turn out large, there would be no monopole problem whatsoever.

Now, if Nature has chosen the option that the lepton number is large enough so that SM symmetry is not restored at high $T$, but without any charge field condensation, even in this case the cosmological consequence would be remarkable, for this would suffice to nonrestore the symmetry in the minimal model of spontaneous CP violation with two Higgs doublets [29]. Namely, without the external charge, in this particular model CP is necessarily restored at high temperature [7] leading to the domain wall problem.

As we mentioned before, it was shown recently that the phenomenon of nonrestoration at high $T$ in the presence of a large charge works in supersymmetry too. We have exemplified our findings on simple $\mathrm{U}(1)$ models [25]. It can be shown that this is true in general, and all that we say above works also in Minimal Supersymmetric Standard Model (MSSM). In this Letter we wish to avoid any model building, but rather concentrate on SM showing that its cosmology may be something entirely different from what one normally imagines.

B. Large $L$ and high $T$ symmetry nonrestoration Let us now discuss in some detail what happens at high temperature if the lepton number is large. Notice first that, since we can assume that the lepton number $L$ is conserved (the sphaleron effects are suppressed [27]), then the ratio of the lepton density $n_{L}$ to entropy density $s$ is constant, too. Now, we are interested in the temperatures above the weak scale when the number of light degrees of freedom grows by another order of magnitude with respect to $T \simeq 1 \mathrm{MeV}$. Thus, the above cited limit $n_{L} / T^{3}<7$ at the time of nucleosynthesis becomes for us an order of magnitude bigger: $n_{L} / T^{3}<70$.

In order to study symmetry breaking, we need to compute the effective potential at high $T$ and high chemical potential. We employ the approximation $\mu_{L}<T$ (where $\mu_{L}$ is the chemical potential associated with the lepton number), since in this case one can obtain the solutions in a closed form. With increased $\mu_{L}$ the physical effect of symmetry breaking gets only stronger [26.

The baryon number of the Universe is negligible, $n_{B} / s \simeq 10^{-11}$, thus we work in the approximation $B=0$. Since the gauge potentials act essentially as the chemical potentials at high $T$, we include them in our $V_{\text {eff }}(T, \mu)$. A word of caution is in order. Although $B=0$, since the quarks carry non trivial baryon number, one must include the associated chemical potential $\mu_{B}$ and we will see below that it does not vanish. We will see that quarks carry a nonvanishing electric charge at high $T$, similarly to the $W$ bosons and the charged Higgs scalar.

Using the techniques of 23,24 the effective potential at high $T\left(T>\mu \gg M_{W}\right)$ and large $n_{L}$ for the Higgs doublet $H$ in the direction of its neutral vacuum expectation value $H=(0, v / \sqrt{2})^{T}$ reads

$$
\begin{aligned}
V_{e f f} & =\lambda \frac{v^{4}}{4}+\frac{g^{2}}{2}\left[\left(A_{0}^{a} A_{0}^{b}\right)\left(A_{i}^{a} A_{i}^{b}\right)-\left(A_{0}^{a} A_{0}^{a}\right)\left(A_{i}^{b} A_{i}^{b}\right)\right] \\
& +\frac{g^{2}}{4}\left[\left(A_{i}^{a} A_{i}^{a}\right)\left(A_{j}^{b} A_{j}^{b}\right)-\left(A_{i}^{a} A_{i}^{b}\right)\left(A_{j}^{a} A_{j}^{b}\right)\right] \\
& -\frac{v^{2}}{8}\left[g^{2}\left(A_{0}^{a} A_{0}^{a}\right)+g^{\prime 2}\left(B_{0} B_{0}\right)\right]+\frac{g g^{\prime}}{2} B_{0} A_{0}^{3} \frac{v^{2}}{2} \\
& +\frac{g^{2}}{4}\left[A_{i}^{1} A_{i}^{1}+A_{i}^{2} A_{i}^{2}\right] \frac{v^{2}}{2}+\mu_{L} n_{L} \\
& -T^{2}\left(\frac{\mu_{L}^{2}}{4}+\frac{\mu_{B}^{2}}{9}\right)+\frac{T^{2}}{3}\left(g^{\prime} B_{0}\right)\left(\mu_{L}-\frac{\mu_{B}}{3}\right) \\
& -\frac{13}{36}\left(g^{\prime} B_{0}\right)^{2} T^{2}-\frac{7}{12} g^{2} A_{0}^{a} A_{0}^{a} T^{2}+\lambda^{\prime} T^{2} \frac{v^{2}}{2} .
\end{aligned}
$$

where $A_{\mu}^{a}$ and $B_{\mu}$ are the $S U(2)_{L}$ and $U(1)_{Y}$ gauge potentials. In the above we have used $g^{\prime} B_{i}=g A_{i}^{3}$ which follows from the equation of motion for $B_{i}$ and

$$
\lambda^{\prime}=\frac{1}{12}\left[6 \lambda+y_{e}^{2}+3 y_{u}^{2}+3 y_{d}^{2}+\frac{3}{4}\left(g^{\prime 2}+3 g^{2}\right)\right],
$$

where $y_{f}$ are the fermionic Yukawa couplings. For simplicity, we take only the third generation of fermions since its couplings are dominant. The inclusion of the first two generations is straightforward and does not change our conclusions.

Notice the point we made before. Although we take $B=0$, the associated chemical potential plays an important role in the above expression. The equations of motion for the gauge fields $A_{\mu}^{a}$ show that the solution discussed in [26] - all gauge potentials zero except for $A_{0}^{3}$ and $A_{1}^{1}$ - is consistent with the above constraints.

Using the constraints $\partial V_{e f f} / \partial x=0$ for $x=\mu_{L}, \mu_{B}$, $g^{\prime} B_{0}$ and $g A_{0}^{3}$ we can rewrite the effective potential as a function of $v$ and $C=\left\langle A_{1}^{1}\right\rangle$ only:

$$
\begin{aligned}
V_{e f f} & =\frac{\lambda}{4} v^{4}+\frac{\lambda^{\prime}}{2} T^{2} v^{2}+\frac{g^{2}}{8} v^{2} C^{2}+\frac{n_{L}^{2}}{T^{2}} \\
& +\frac{4 n_{L}^{2}\left(3 v^{2}+12 C^{2}+14 T^{2}\right)}{54 v^{2} C^{2}+\left(87 v^{2}+96 C^{2}\right) T^{2}+112 T^{4}} .
\end{aligned}
$$

The effective potential is manifestly bounded from below and it is a simple exercise to minimize it. We work with a small $\left(H^{\dagger} H\right)^{2}$ coupling - only for the sake of presenting simple analytic expressions. We find the results presented below. In discussing them, it will turn out useful to have the individual distribution of the various charges. Namely, here lies an important point that was overlooked before [26] and that plays a significant role for our considerations about the monopole problem, i.e. the fact that quarks, the charged Higgs and $W$ carry electromagnetic charge in spite of having lepton number zero. Since the $\mu$-dependent part of the effective potential can be written 23, 24

$$
V_{e f f}^{(\mu)}=-\frac{T^{2}}{12} \sum_{f} \mu_{i}^{2}-\frac{T^{2}}{6} \sum_{b} \mu_{i}^{2}-\sum_{b} \mu_{i}^{2}\left|\phi_{i}\right|^{2}+\mu_{L} n_{L},
$$


one can find for the distribution of fermionic and bosonic charges

$$
\begin{aligned}
& \left(\mathcal{Q}_{F}^{a}\right)_{i}=q_{i}^{a} \mu_{i}\left(\frac{T^{2}}{6}\right), \\
& \left(\mathcal{Q}_{B}^{a}\right)_{i}=q_{i}^{a} \mu_{i}\left(\frac{T^{2}}{3}+2\left|\phi_{i}\right|^{2}\right),
\end{aligned}
$$

where $q_{i}^{a}$ denote the transformation property $\varphi_{i} \rightarrow$ $e^{i q_{i}^{a} T^{a}} \varphi_{i}, \varphi_{i}$ stands for any field, $a$ goes over all the relevant charges $\left(L, B, Y / 2, T_{3 W}\right)$ and $\mu_{i}=\sum_{a} q_{i}^{a} \mu_{a}$.

Let us first shortly discuss the case of small lepton asymmetry or, more precisely, $n_{L}<\left(n_{L}\right)_{1} \equiv$ $(4 / 3) \sqrt{\lambda^{\prime}} T^{3}$. In such a case only the trivial solution is possible: $v=C=0$. This is the usual scenario of small charge densities.

It is an easy exercise to compute the distribution of charges. One finds $L\left(\nu_{L}\right)=L\left(e_{L}\right)=\frac{3}{8} L$ and $L\left(e_{R}\right)=\frac{2}{8} L$ for the $L$ number distribution (notice that $L\left(\nu_{L}\right)=L\left(e_{L}\right)$ since $S U(2)$ is not broken). For the electromagnetic charge (we list only the nonvanishing ones)

$$
\begin{aligned}
Q\left(e_{L}\right) & =-\frac{3}{8} L, Q\left(e_{R}\right)=-\frac{2}{8} L, \\
Q\left(u_{R}\right) & =\frac{2}{8} L, Q\left(d_{R}\right)=\frac{1}{8} L, Q\left(h^{+}\right)=\frac{2}{8} L,
\end{aligned}
$$

so that $Q_{t o t}=0$ as it should be, but the charge is distributed among both fermions and charged Higgs bosons (we find later $W^{ \pm}$participating too).

Let us now focus on the following intermediate range $\left(n_{L}\right)_{1}<n_{L}<\left(n_{L}\right)_{2} \equiv\left(n_{L}\right)_{1}\left(1+\frac{203}{192} g^{2} / \lambda^{\prime}\right)$. It is easy to show that now $v \neq 0$, but $C$ still vanishes

$$
\begin{aligned}
V_{e f f}(v \neq 0) & =V_{e f f}(v=0)-\frac{21}{58 T^{2}}\left[n_{L}-\left(n_{L}\right)_{1}\right]^{2}, \\
v^{2} & =\frac{112}{87} \frac{n_{L}-\left(n_{L}\right)_{1}}{\left(n_{L}\right)_{1}} T^{2}, \quad C=0 .
\end{aligned}
$$

Clearly, $S U(2)_{L} \times U(1)_{Y}$ is spontaneously broken down to $U(1)_{e m}$, but there is no condensation of $W$ bosons. This means that for such values of the lepton number it is energetically preferable for the system to cancel the electric charge by means of the asymmetries in the quarks and charged Higgs boson, but no spontaneous breaking of electromagnetism takes place.

Finally, let us consider the case of large lepton asymmetry, $n_{L}>\left(n_{L}\right)_{2}$. Now, on top of the Higgs mechanism, we have also the $W$ condensation [26]: $C \neq 0$. Notice that $\left(n_{L}\right)_{2}$ depends very mildly on the Higgs mass in the physically interesting range between $80 \mathrm{GeV}$ and 500 GeV: $\left(n_{L}\right)_{2} \approx(2.0-2.5) T^{3}$. This is clearly much below the upper limit $70 T^{3}$. Strictly speaking, for $n_{L}>\left(n_{L}\right)_{1}$ we have $\mu_{L}>T$ so that our analytic formulae are not exact. Thus, we have also performed numerical computations for the case of large chemical potentials and finite $\lambda$, which prevents exact analytic results. This amounts to including the terms in the effective potential of the order of $\mu^{4}$. Our findings from this standard procedure are shown in the table below, where we give the corrections to the critical densities calculated analytically.

\begin{tabular}{|c|c|c|c|}
\hline$m_{H}(\mathrm{GeV})$ & $\left(n_{L}\right)_{1}^{\text {num }} /\left(n_{L}\right)_{1}$ & $\left(n_{L}\right)_{2}^{\text {num }} /\left(n_{L}\right)_{2}$ & $v_{2}^{\text {num }} / v_{2}$ \\
\hline 100 & 1.05 & 1.28 & 1.01 \\
\hline 200 & 1.07 & 1.34 & 0.92 \\
\hline 400 & 1.13 & 1.52 & 0.96 \\
\hline 600 & 1.21 & 1.66 & 1.06 \\
\hline
\end{tabular}

Table 1: The ratios between the exact numerical solutions (numerators) and the approximate analytic solutions (denominators) described in the text as functions of the Higgs mass. $v_{2}^{(\text {num })}$ is the Higgs vev for $n_{L}=\left(n_{L}\right)_{2}^{(\text {num })}$.

Clearly, the numerical study confirms our analytical findings of symmetry breaking for large densities. Although the precise value of the second critical density (the first critical density is almost unchanged) is increased about $30 \%$ for a reasonable values of the Higgs mass, this does not affect the possibility of symmetry restoration. Namely, the critical density remains still an order of magnitude below the allowed value of $70 T^{3}$.

C. The consequences: monopole and domain wall problems. We have seen that a large enough lepton density implies symmetry breaking at high temperature, which opens the door for the solution of the monopole problem. The simplest possibility is to follow the scenario 17] for the high $\mathrm{T}$ breaking of $U(1)_{\mathrm{em}}$. The essential point here is that if the $U(1)_{e m}$ symmetry is broken due to a large external charge, it would be broken for the whole parameter space of the theory and for all temperatures above $M_{W}$ all the way to the GUT scale. Thus, monopoles may never be created and there would obviously be no problem at all. Even if they did get produced they would surely have time to annihilate. In this sense it is only our scenario that guarantees the solution to the monopole problem. Of course one must make sure that $U(1)_{e m}$ is really broken. If we restrict ourselves to the $\mathrm{SM}$ and work in the regime of $W$-condensation, it is not clear to us what the precise situation is. First of all, the fact that the $W$ has condensed implies the breakdown of the rotational invariance and the description of the formation, if any, of the monopoles at the GUT scale might be completely different from the usual one. Secondly, if monopoles do get formed, they might not annihilate rapidly enough or might not annihilate at all due to the anti-screening effects of the $W$-background. These issues are extremely important and certainly deserve a separate investigation. We would like to point out, however, that the situation is more transparent if we consider a simple extension of the SM where an electrically charged field $S$ is present (a similar extension would be to add another doublet). In a grand unified theory this singlet would be embedded in a larger representation, such as an $S U(5) \underline{10}$ (a doublet would belong to another $\underline{5}$ ). The idea of one singlet in addition to the SM Higgs was already pursued 
in [18], [19]. However, as we axplained in the introduction, without the external charge this mechanism may not work 20], 21.

We have explicitly checked that, for large enough lepton number, the SM gauge group is broken at high $T$. Moreover, since the field $S$ gets a VEV, $U(1)_{e m}$ is spontaneously broken. More important, similarly to what we have described before for the SM, there exists a range of values of the lepton number for which the $W$-condensation does not take place. Under these conditions, the monopole problem is solved. Namely, at $T \simeq M_{X}$ when the GUT symmetry (say $S U(5)$ ) breaks down, $U(1)_{e m}$ is broken and there will be no creation of monopoles. It is intriguing that a realistic realization of this idea may take place within the Minimal Supersymmetric Standard Model (MSSM) where charged Higgs fields are present. The only price to pay is to accept the idea that the lepton number may be large enough. Once this step is made, the monopole problem is no longer with us. This is a remarkable result.

What about the domain wall problem? Clearly, the presence of large lepton number asymmetry through the nonrestoration at high temperature solves the domain wall problem in an analogous manner. For example, this would solve the well-known domain wall problems associated with the spontaneous violation of $\mathrm{CP}$ 229] or the $Z_{2}$ natural flavour conservation symmetry [30].

D. Summary and Outlook We have argued that a large lepton asymmetry in the Universe may mean an automatic solution of the monopole and domain wall problems through symmetry nonrestoration at high T. As far as the monopole problem is concerned, this idea works for simple extensions of the SM and in particular in the MSSM.

For all we know the lepton number of the Universe may be comparable, if not bigger than, the entropy of the Universe. The fact that the large lepton number can be consistent with the small baryon number in the context of grand unification has been pointed out a long time ago [31] and recently a model for producing large $L$ and small $B$ has been presented [32]. We stress, however, that our findings should remain valid if, instead of the lepton number we consider any other conserved charge in the system under consideration.

We would like to thank G. Dvali, M. Shaposhnikov and G.G. Ross for interesting discussions. This work was partially supported by the British Royal Society and by the Ministry of Science and Technology of Slovenia (B.B.) and by EEC under the TMR contract ERBFMRXCT960090 (G.S.). This work was completed during the Extended Workshop on the Highlights in Astroparticle Physics, held at ICTP from October 15 to December 15, 1997. B.B. and A.R. thank ICTP for hospitality during the course of this work.
[1] J. Preskill, Phys. Rev. Lett. 43, 1365 (1979).

[2] Ya.B.Zeldovich, I.Yu.Kobzarev, and L.B.Okun, JETP 40, 1 (1974).

[3] G. Dvali, H. Liu and T. Vachaspati, Phys. Rev. Lett. 80, 2281 (1998).

[4] S.Weinberg, Phys. Rev. D9, 3357 (1974).

[5] R.N. Mohapatra and G. Senjanović, Phys.Rev. Lett. 42, 1651 (1979); Phys. Rev.D20, 3390 (1979).

[6] G. Dvali and G. Senjanović, Phys. Rev. Lett. 74, 5178 (1995).

[7] G. Dvali, A. Melfo, and G. Senjanović, Phys. Rev. D54, 7857 (1996).

[8] G. Dvali, A. Melfo, and G. Senjanović, Phys. Rev. Lett. 75, 4559 (1995).

[9] G. Bimonte and L. Lozano, Nucl.Phys. B460, 155 (1996).

[10] G. Bimonte, D. Iniguez, A. Tarancon and C. L. Ullod, hep-lat/9802022.

[11] K. Jansen and M. Laine, hep-lat/9805024.

[12] T. G. Roos, Phys. Rev. D54, 2944 (1996); G. AmelinoCamelia, Phys. Lett. B388, 776 (1996); M. Pietroni, N. Rius and N. Tetradis, Phys. Lett. B397, 119 (1997); J. Orloff, Phys. Lett. B403, 309 (1997).

[13] H. Haber, Phys. Rev. D 46, 1317 (1982).

[14] M. Mangano, Phys.Lett. 147B, 307 (1984).

[15] G. Dvali and K. Tamvakis, Phys. Lett. B378, 141 (1996).

[16] B. Bajc, A. Melfo, and G. Senjanović, Phys. Lett. B387, 796 (1996); B. Bajc and G. Senjanović, Nucl. Phys. Proc. Suppl., 52A, 246 (1997).

[17] P. Langacker and S.-Y. Pi, Phys. Rev. Lett. 45, 1 (1980).

[18] V. D. Dixit and M. Sher, Phys. Rev. Lett. 68, 560 (1992).

[19] T. H. Farris, T. W. Kephart, T. J. Weiler and T. C. Yuan, Phis. Rev. Lett. 68, 564 (1992).

[20] E. Gates, L. M. Krauss and J. Terning, Phys. Lett. B284, 309 (1992).

[21] R. Holman, T. W. B. Kibble and S.-J. Rey, Phys. Rev. Lett. 69, 241 (1992).

[22] A. D. Linde, Phys. Rev. D14, 3345 (1976).

[23] H. E. Haber and H. A. Weldon, Phys. Rev. D25, 502 (1982).

[24] K. M. Benson, J. Bernstein, and S. Dodelson, Phys. Rev. D44, 2480 (1991).

[25] A. Riotto and G. Senjanović, Phys. Rev. Lett. 79, 349 (1997).

[26] A. D. Linde, Phys. Lett. 86B, 39 (1979).

[27] J. Liu and G. Segre, Phys. Lett. B338, 259 (1994).

[28] H.-S. Kang and G. Steigman, Nucl. Phys. B372, 494 (1992), and references therein.

[29] T. D. Lee, Phys. Rev. D8, 1226 (1973).

[30] S. L. Glashow and S. Weinberg, Phys. Rev. D15, 1958 (1977).

[31] J. A. Harvey and E. W. Kolb, Phys. Rev. D24, 2090 (1981).

[32] A. Casas, W.-Y. Cheng, and G. Gelmini, hep -ph/9709289. 